\title{
On the kinematic interpretation of cosmological redshifts
}

\author{
G.Ter-Kazarian* \\ Byurakan Astrophysical Observatory, 378433, Aragatsotn District, Armenia
}

\begin{abstract}
We describe what is essentially a correct solution to the kinematic interpretation of cosmological redshifts in standard cosmological model. In the framework of 'stretching of space' point of view of standard cosmological model, we study the 'lookforward' history of expanding universe, subject to certain rules, in order to define the kinetic recession velocity of luminous source along the observer's line-of-sight in unique way, straightforwardly in terms of cosmological redshift. In doing this, we use an alternative way of separating the spectral shifts into infinitesimally displaced 'relative' spectral bins between adjacent emitter and absorber consequent on expansion of the universe, measured at infinitesimally separated space-time points, and sum over them to overcome the ambiguity which presents the parallel transport of four-velocity of source to an observer in the Robertson-Walker curved space-time. The crux of our solution - the kinetic recession velocity of comoving astronomical object, is always subluminal even for large redshifts of order one or more, so that it does not violate the fundamental physical principle of causality. Our analysis establishes the ubiquitous relationship of overall cosmological redshift and kinetic recession velocity, which is utterly distinct from a familiar global Doppler shift formula. A difference of global Doppler velocity and kinetic recession velocity, for redshifts $0.9 \leq z \leq 800$, is $\geq 0.072 c$, where a maximum value, $0.187 c$, is reached at redshifts $z=4.5-5.1$. In particular case of such an implementation along the null geodesic, we show that the kinetic recession velocity is reduced to a well known global Doppler velocity. We discuss the implications for the case of a zero-density cosmological model of Milne universe, whereas a correspondence to the more usual special relativity notion of relative speed retains. In Table 1 , we are summing up kinetic recession velocities of some typical distant astronomical objects with spectroscopic redshift determinations collected from the literature.
\end{abstract}

Keywords: galaxies: high-redshift-galaxies: distances and redshifts_cosmology: theory

\section{Introduction}

A complex study of more distant astronomical objects raises several disturbing questions of the physical interpretation of cosmological dynamics, see e.g. (Bolós \& Klein, 2012, Bunn \& Hogg, 2009, Chodorowski, 2011, Davis \& Lineweaver, 2004, Emtsova \& Toporensky, 2019, Grøn \& Elgarøy, 2007, Harrison, 1993, 1995, 2000, Kaya, 2011, Klein \& Collas, 2010, Klein \& Randles, 2011, Liebscher, 2007, Murdoch, 1977, Narlikar, 1994, Peacock, 1999, 2008, Peebles, 1993, Peebles et al., 1991, Pössel, 2019, Silverman, 1986, Stuckey, 1992, Whiting, 2004).

The astronomers for decades routinely do not distinguish between the Hubble's empirical linear 'redshift-distance' law, $c z=H L$ and the linear 'velocity-distance' law, $\dot{L}=H L$, derived later on theoretical basis, where and throughout the overdot represents differentiation with respect to epoch synchronous time, $t, z$ is the redshift, $L$ is the proper distance from a galaxy to an observer at epoch $t$, and $H$ is the Hubble's parameter. They express redshifts as if they were radial velocities to convert cosmological redshifts into velocity. But aside from the observations for relatively nearby galaxies, for later measurements of more distant objects, with redshifts of order one or more, a special relativity (SR) Doppler interpretation is neither useful nor adequate. One should, therefore, drop this interpretation in favor of the 'stretching of space' point of view of general relativity (GR) description with a

*gago_50@yahoo.com 
dynamical space. Such a concept of 'stretching of space' has by itself no physical content, but it is merely the choice for analysing phenomena. In accord to it, the peculiar velocity of the object with respect to the Hubble flow declines in magnitude as the universe expands $\delta v \propto 1 / R(t)$, where $R(t)$ is the scale factor, and thus, at $t \rightarrow \infty$, the peculiar velocity tends to zero leaving the object moving with the Hubble flow. This immediately has fostered a startling view to think of present day values of the rate of expansion $\dot{L}(t, z)$ as the so-called 'proper' recession velocity of comoving galaxy of redshift $z$ away from an observer. We should look very sceptically at any argument which uses or implies the concept of superluminal 'proper' recession velocity as a real physical velocity, as if the meaning of that were clear and obvious. Although it is extremely hard to envisage a consistent theory having such a logical impossibility of superluminal 'proper' recession velocity, this problem stood open for nearly a century as a startling preoccupation of wide community of astronomers, see e.g. (Davis \& Lineweaver, 2004, Lineweaver \& Davis, 2005). There is an important reason to question the validity of such a description of apparent superluminal growth of the universe, because we inevitably encounter with a crucial question of "what is a practical measure of being swept up of galaxy in expanding universe?"

Our primary interest in this article is rather to focus on the principle issue of how to reconcile the cosmological interpretation of redshifts with the most natural kinematic interpretation. We try to impart some knowledge about the physical nature of the kinetic velocity of luminous source along the observer's line-of-sight in unique way, straightforwardly in terms of cosmological redshift, in a mostly non-technical, nevertheless hopefully precise and consistent language. The solutions given in present article demonstrate its advantage over the specific extant definitions in the cited literature.

- We solve startling difficulties of superluminal 'proper' recession velocities in standard cosmological model, which is the principle issue for the physics. This peculiarity deserves careful study, because it furnishes valuable theoretical clues about the interpretation of kinetic velocity of luminous object relative to observer in GR, a systematic analysis of properties of which happens to be surprisingly difficult by conventional methods. Avoiding from any mistakes, therefore, we preferred to work in an infinitesimal domain. The problem of subjecting the four-velocity vector of the luminous source to parallel transport will not be broached in this paper, though it is hoped that the present formulation of the theory will facilitate the task. We advocate with socalled 'lookforward' history of expanding universe, to achieve an unique definition of the kinetic recession velocity of astronomical object in terms of redshift. At any rate, it is remarkable that these definitions above completely determine this velocity to always be subluminal even for large redshifts of order one or more, and thus, it does not violate the fundamental physical principle of causality. This will help astronomers to derive measurable quantities for further study of the problems of fundamental physics of early universe.

- We discuss the implications of this approach for two instructive cases: (i) We show that a derived general solution is reduced to global Doppler shift along null geodesic. (ii) For the limit of a zero-density cosmological model of Milne universe, a correspondence to the more usual special relativity notion of relative speed retains.

- We give (App.A) a reappraisal in a deep way of the preliminary attempts of 'standard' kinematic interpretation of redshifts as accumulated Doppler-shifts consequent on recession, widely discussed in literature. Its study is valuable as affording insight into the whole subject. In doing this, we are not suggesting any doubt about the principle statement. Rather, we doubt the method of calculations, which as we have shown are in error. Moreover, this statement is a crux of our derivation of an essentially correct solution to a kinematic interpretation of cosmological redshifts.

Regarding a generalization, in the original sense of the term, of relative velocity of luminous source in a general Riemannian space-time, indeed this problem is the most important for GR. It will be separate topic for an investigation elsewhere. 
With this perspective in sight, we will proceed according to the following structure. To start with, Section 2 deals with a startling challenge of the superluminal recession velocities. Deriving in Section 3 the kinetic recession velocity of a distant astronomical object, we reconcile the cosmological interpretation of redshift with the correct solution to a kinematic interpretation of redshifts as accumulated Doppler-shifts. In Subsection 3.1, we show that a general solution is reduced to a global Doppler shift along the null geodesic. In Section 4, we give a brief outline of a cosmological toy model of the Friedmann-Robertson-Walker (FRW)-universe for zero-density in the RW coordinates. Concluding remarks are given in Section 5. A few more technical details in use are deferred to appendices. Appendix A provides a brief critical discussion of some key objectives with the analysis aimed at clarifying the current situation of the often met preliminary attempts of kinematic interpretation of redshifts as accumulated Doppler-shifts. This illustrates the problems and also hint at a possible solution. It was used as a backdrop to explore in Section 3 the kinetic recession velocity. Since many of the issues discussed in this contribution are conceptual, the observational status of these concepts is important. In Appendix B, therefore, we calculate the kinetic recession velocities of some typical distant astronomical objects with spectroscopic redshift determinations collected from the literature, which are listed in Table 1.

\section{The concerns of the superluminal recession velocities}

In past decades, the debate about superluminal 'proper' recession velocities was less fettered by observational evidence for large redshifts, but it gathers support from a breakthrough made in recent observational efforts, and at present it would require a good deal more ingenuity, which is of immense significance for the foundation of GR. Today there is no known feasible alternative way to account for credible explanation of the principle problem of superluminally receding galaxies. Such claims cannot be accepted as a convincing ones. This belief is suspect, and should be critically re-examined. A healthy degree of scepticism based on at least three objections is in order:

- The conclusions derived from assertion that the object is moving with the 'relative' velocity, $\dot{L}(t, z)$, to an observer must be treated with caution. Indeed, the incredibility of such an inference has been greatly enhanced by the recall that GR provides no a priori definition of 'relative' velocity, because their velocities are vectors at different events. This inability to compare vectors at widely separated space-time events was the fundamental feature of a curved space-time. Different coordinate reference frames and notions of 'relative' velocity yield different results for the motion of distant test particles relative to a particular observer. Bolós (2006, 2007), Bolós \& Klein (2012), Bolós et al. (2002), Klein \& Collas (2010), Klein \& Randles (2011) address the question of relative velocities in GR. Consequently, three distinct coordinate charts, each with different notions of simultaneity, are employed by Bolós \& Klein (2012) in the calculations of the four geometrically defined inequivalent concepts of relative velocity: Fermi, kinematic, astrometric, and the spectroscopic relative velocities. These definitions of relative velocities depend on two different notions of simultaneity: 'spacelike simultaneity' (or 'Fermi simultaneity') (Klein \& Randles, 2011, Walker, 1935) as defined by Fermi coordinates of an observer, and 'lightlike simultaneity' as defined by optical (or observational) coordinates of an observer (Ellis, 1985). The Fermi and kinematic relative velocities can be described in terms of the 'Fermi simultaneity', according to which events are simultaneous if they lie on the same space slice determined by Fermi coordinates. Thereby, for an observer following a timelike worldline in Riemannian space-time, Fermi-Walker coordinates provide a system of locally inertial coordinates. If the worldline is geodesic, the coordinates are commonly referred to as Fermi or Fermi normal coordinates. Useful feature of Fermi coordinates was that the metric tensor expressed in these coordinates is Minkowskian to first order near the path of the Fermi observer, with second order corrections involving only the curvature tensor (Manasse \& Misner, 1963). Klein \& Randles (2011) find explicit expressions for the Fermi coordinates for Robertson-Walker (RW) spacetimes and show that the Fermi chart for the Fermi observer in non-inflationary RW space-times is global. However, rigorous results for the radius of a tubular neighborhood of a timelike path for the domain of Fermi coordinates are not available. The spectroscopic (or barycentric) and 
astrometric relative velocities, which can be derived from spectroscopic and astronomical observations, mathematically, both rely on the notion of light cone simultaneity. According to the latter, two events are simultaneous if they both lie on the same past light cone of the central observer. It is shown that the astrometric relative velocity of a radially receding test particle cannot be superluminal in any expanding RW space-time. Necessary and sufficient conditions are given for the existence of superluminal Fermi speeds. Note that for the Hubble velocity, the proper distance is measured along non geodesic paths, while for the Fermi velocity, the proper distance is measured along spacelike geodesics. In this respect the Fermi velocity seems to be more natural, but the Hubble velocity is defined at all space-time points, whereas the Fermi velocity makes sense only on the Fermi chart of the central observer. Although alluded four definitions of relative velocities have own physical justifications, all they are subject to many uncertainties, and the ambiguity still remains.

- What is more, the 'proper' recession velocity, $\dot{L}(t, z)$, referred to in this claim is an unnatural quantity, because specifically it is the rate of change of the proper distance to the object with respect to the cosmic time coordinate, as measured at the present cosmic time. It has nothing to do with the object at all existed in the past. This coordinate velocity is a mere artifact to discuss, because it refers to events far outside of an observer's light cone.

- The picture of expanding universe is fully consistent with SR locally and GR globally (Robertson, 1935, Walker, 1936). One may, therefore, consider the large enough distance characteristic of the universe as a whole only within a theoretical framework capable of dealing with velocities approaching that of light. Any correctly defined relative velocity should be less than the speed of light, independent of any distance or time lag. The important reason to question the validity of a prediction of superluminal recession velocities, which became untenable, is the fact that it violates the fundamental principle of causality within these frameworks. As Hu et al. (1993) assert, "Superluminal expansion might be most naturally defined as that where any two comoving points eventually lose causal contact." Let us put aside subtleties of RW-metric of expansion (or whatever) of curved space, and focus on a clear academic question whether it is allowed for particle to attain superluminal velocity in this space with the given metric (g), where the particle always resides on the mass shell:

$$
p^{2}=g_{\mu \nu} p^{\mu} p^{\nu}=m_{0}^{2} c^{4},
$$

provided, $p^{\mu}(E, c \vec{p})$ are the components of 4-momentum. But the truth is the contrariwise: situating on the mass shell, the particles cannot attain the velocities exceeding the speed of light even after making due allowances for bringing one back in time to an epoch when the universe was very young. A reliable way to see that the prediction of superluminal velocities (recession or whatever) is in error is the 'monad' formulation of metric theory of GR. The latter is the mathematical apparatus of physically observable quantities (Cattaneo, 1958, Eckart, 1940, Leaf, 1951, Pirani, 1962, Zelmanov, 1944, 1976). Monad formalism in the terms of Cartan's external calculus is worked out by Massa (1974). In this framework, one chooses a suitable family of observers based on the definition of 'congruence' of time-like world lines in given region. Whereas the tangent unit vector $\tau^{\mu}$ ('monad') of 'congruence' time-like world line is $\tau^{\mu} \equiv u^{\mu}=d x^{\mu} / d s$, incorporated with the normalization condition $\tau^{\mu} \tau_{\mu}=\tau^{\mu} \tau^{\nu} g_{\mu \nu}=1$. The metric tensor can be written in the form $g_{\mu \nu}=\tau_{\mu} \tau_{\nu}-h_{\mu \nu}$, where $h_{\mu \nu}$ is the metric tensor of local 3D spatial section of an observer orthogonal to unit vector $\tau^{\mu}: \tau^{\mu} h_{\mu \nu}=\tau^{\mu} h_{\mu}^{\nu}=\tau_{\mu} h_{\nu}^{\mu}=\tau_{\mu} h_{\nu}^{\mu}=$ $\tau_{\mu} h^{\mu \nu}=\tau_{\mu}\left(\tau^{\mu} \tau^{\nu}-g^{\mu \nu}\right)=0$. Then, instead of an arbitrary unobservable local displacement $d x^{\mu}$, usually, the observable standards of spatial, $\widetilde{d x^{\nu}}=-h_{\mu}^{\nu} d x^{\mu}$, and time, $d \tau=\tau_{\mu} d x^{\mu}$, intervals can be introduced for whatever metric, so that the temporal and spatial components of tensors are clearly separated. Going to a new time coordinate, $\tau$, one sets the potentials to zero at the world-point one is considering. The line element should be $d s=c d \tau \sqrt{1-v^{2} / c^{2}}$, where $v^{2}=h_{\mu \nu} v^{\mu} v^{\nu}$, and $v^{\mu}=-h_{\nu}^{\mu}\left(d x^{\nu} / d \tau\right)$ are the spacial components of particle velocity, so that the 4-dimensional speeds can be expressed through 3-dimensional, $u^{\mu}=\left(\tau^{\mu}+v^{\mu} / c\right) / \sqrt{1-v^{2} / c^{2}}$. The 'congruence' of time-like world lines of the reference frame are characterized by four scalars, 
subject to certain rules (Vladimirov, 1982), respectively: (i) the 'first curvature', $R_{1}$, defined by relation $R_{1}^{2}=-F_{\mu} F^{\mu}$, where $F_{\mu}$ is the chr.inv.-vector of the 'acceleration of the instrument of reference frame' ('gravitational inertial force'), (ii) 'stretching', $\varepsilon$, defined as $\varepsilon=-(1 / 2) D$, where $D_{\mu \nu}$ is the chr.inv.-tensor of the 'velocities of deformation of space', (iii) the 'rotation', $\Omega$, defined by relation $\Omega^{2}=(1 / 2) A_{\mu \nu} A^{\mu \nu}$, where $A_{\mu \nu}$ is the chr.inv.-tensor of the 'angular velocity of the rotation of space' due to its non-holonomity (the non-orthogonality of the time lines to the spatial section), and (iiii) 'shear', $\sigma$, of congruence defined as $\sigma^{2}=(1 / 2)\left(D_{\mu \nu} D^{\mu \nu}-\varepsilon^{2} / 2\right)$. The 'monad' method is most effective in special systems of coordinates, so-called 'chronometric' $\left(\tau^{i}=d x^{i} / d s\right)=0$, chosen so that the congruence of coordinate lines $x^{0}\left(x^{i}=\right.$ const $)$ coincides with the congruence of time-like world lines of the reference system $\tau$. Solving the normalization condition $\tau^{\mu} \tau^{\nu} g_{\mu \nu}=1$ the 'monad' is calibrated $\tau^{\mu}=g_{0}^{\mu} / \sqrt{g_{00}}$. The condition of coincidence of the congruence $\tau$ and $x^{i}=$ const defines a whole class of 'chronometric' coordinate systems linked to each other by special "chronometric" coordinate transformations, which are found from condition $\tau^{i}=0$ in all chronometric coordinate systems. The physically observable (projected) quantities are invariant under "chronometric" coordinate transformations, and called 3-tensors or "chronometric invariants" (chr.inv.), which are invariant in the spatial section of the observer. So that the dispersion relation (1) can be recast into the form

$$
p^{2}=E^{2}-c^{2} \vec{p}^{2}=E^{2}-c^{2} h_{\mu \nu} \bar{p}^{\mu} \bar{p}^{\nu}=m_{0}^{2} c^{4},
$$

provided, $\bar{p}^{\mu}=-m_{0} c h_{\alpha}^{\mu}\left(d x^{\alpha} / d s\right)=m v^{\mu}$ are the components of chr.inv.-vector of 3-momentum, and $m=m_{0} c \tau_{\alpha} d x^{\alpha} / d s=m_{0} / \sqrt{1-v^{2} / c^{2}}$ is the chr.inv.-invariant of moving (relativistic) particle dynamical mass. In no sense, therefore, can GR be said to allow for particle to attain superluminal velocity, and hence the vacuum value of a velocity of light is the universal maximum attainable velocity of a material body found in this space regardless of coordinate reference frame. Thus, the 'monad' formulation of GR ruptures once and for all the claim of separation between two more distant objects to increase faster than the speed of light as it has insufficient dimensions. The above said appears to provide a new perspective to have met the challenge of superluminal recession velocities, which the conventional scenario of expanding universe of standard cosmological model presents. In some instances, the distant astronomical objects are observed to exhibit redshifts in excess of unity (earlier epochs), thus, only a consistent theory would fill the void to tackle the key problems of a dynamics of such objects.

\section{The kinetic recession velocity of comoving astronomical object in RW space-time}

In the framework of standard cosmological model, one assumes that the universe is populated with comoving observers. In the homogeneous, isotropic universe comoving observers are in freefall, and obey Wayl's postulate: their all worldlines form a 3-bundle of non-intersecting geodesics orthogonal to a series of spacelike hypersurfaces, called comoving hypersurfaces. In case of expansion, all worldlines are intersecting only at one singular point. The clocks of comoving observers, therefore, can be synchronized once and for all. Let the proper time, $t$, of comoving observers be the temporal measure. Suppose $R(t)$ is the scale factor in expanding homogenous and isotropic universe. One considers in the so-called cosmological rest frame a light that travels from a galaxy to a distant observer, both of whom are at rest in comoving coordinates. As the universe expands, the wavelengths of light rays are stretched out in proportion to the distance $L(t)$ between co-moving points $\left(t>t_{1}\right)$, which in turn increase proportionally to $R(t)$ (Harrison, 1993, 1995):

$$
\frac{\lambda(t)}{\lambda\left(t_{1}\right)}=\frac{d t}{d t_{1}}=\frac{R(t)}{R\left(t_{1}\right)}=\frac{L(t)}{L\left(t_{1}\right)} .
$$

Reviewing notations, $L_{1} \equiv L\left(t_{1}\right)$ is the proper distance to the source at the time when it emits light, $L(t)$ is the same distance to the same source at light reception. Thus, the photons are seen as permanently loosing energy due to being cosmologically redshifted, because of which in modern cosmology 
the photons are usually taken as negligibly influencing the present-day expansion dynamics. Such confidence is somehow based on the undoubted successes of GR in our immediate cosmic surroundings, with or without a $L$-term which has been termed 'dark energy' when it has the sign opposite to that of energy, whose necessity is still debated.

In what follows, we are going to define a more rigorous viable concept of kinetic recession velocity of a comoving distant galaxy of redshift $z$, which crossed past light cone at time $t_{1}$, at point $\left(A_{1}\right)$ away from comoving observer $(O)$ at the present time $t$. This is rather technical topic, and it requires care to do correctly. To clarify the issues further, it should help a few noteworthy points of Fig. 1, which illustrates the lookforward history of expanding homogenous and isotropic universe. The principle

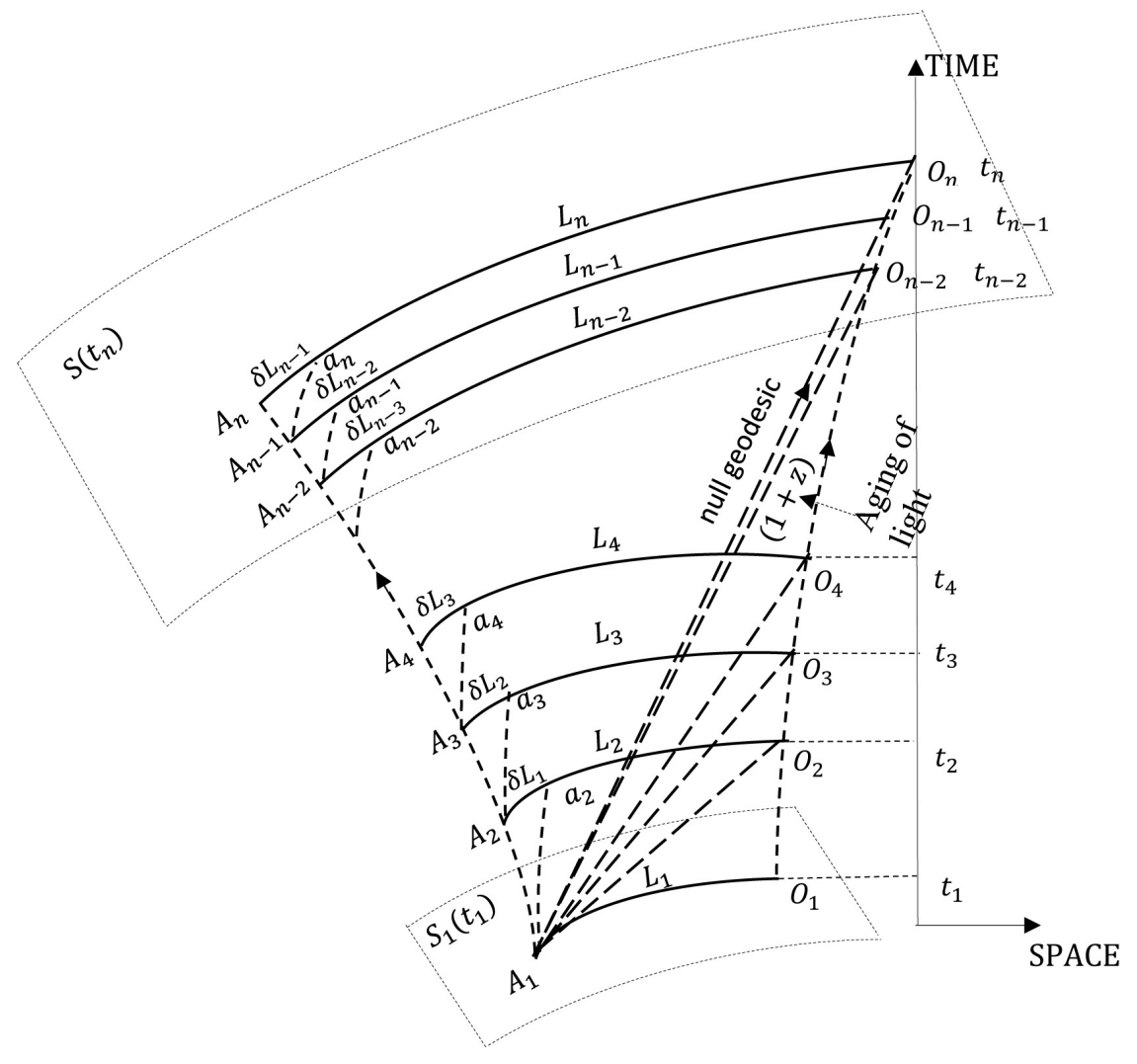

Figure 1. "Lookforward" history of expanding homogenous and isotropic universe: The increase of the proper distance $L_{i}$ between a galaxy $\left(A_{i}\right)$ and observer $\left(O_{i}\right)$ (at epoch $t_{i}$ ) is viewed over different epochs $(i=1,2, \ldots, n)$, with the infinitesimal time difference $\left(\left(t_{i}-t_{i-1}\right) \rightarrow 0\right)$. Whereas $t_{n} \equiv t$ and $L_{n} \equiv L(t)$. An observer $\left(O_{i}\right)$ in its rest frame of reference measures the frequency of light rays emitted by a galaxy $\left(A_{i}\right)$ viewed over different epochs $(1, \ldots, i)$ of expansion. Each proper distance $L_{A_{i-1} O_{i-1}}\left(t_{i-1}\right)$ (at epoch $t_{i-1}$ ) is identically mapped on the line segment $L_{a_{i} O_{i}}\left(t_{i}\right)$ of proper distance (at infinitesimally close epoch $t_{i}$ ), such that $L_{a_{i} O_{i}}\left(t_{i}\right) \equiv L_{A_{i-1} O_{i-1}}\left(t_{i-1}\right)$. Proper distance is the spatial geodesic measured along a comoving hypersurface $S\left(t_{i}\right)$ of constant cosmic time, into which a natural foliation of the space-time is defined by the RW metric. Null geodesic of a light signal from a galaxy $\left(A_{1}\right)$ to the observers $O_{i}\left(O_{n} \equiv O\right)$ is also plotted.

foundation of our approach comprises the following steps. Let $L_{i} \equiv L\left(t_{i}\right)$ be the 'proper distance' between a galaxy $\left(A_{i}\right)$ and observer $\left(O_{i}\right)$, at given epoch $\left(t_{i}\right)$, while the increment of the $L_{i}$ is viewed over different epochs $(i=1,2, \ldots, n)$, with the infinitesimal time difference $\left(\left(t_{i}-t_{i-1}\right) \rightarrow 0, n \rightarrow \infty\right)$. Whereas $t_{n} \equiv t$ and $L_{n} \equiv L(t)$. We assume that an observer $\left(O_{i}\right)$ in its rest frame of reference measures the frequency of light rays emitted by a galaxy $\left(A_{i}\right)$, viewed over different epochs $(1, \ldots, i)$ of expansion. Each proper distance $L_{A_{i-1} O_{i-1}}\left(t_{i-1}\right)$ (at epoch $\left.t_{i-1}\right)$ is identically mapped on the line segment $L_{a_{i} O_{i}}\left(t_{i}\right)$ of proper distance (at infinitesimally close epoch $t_{i}$ ), such that $L_{a_{i} O_{i}}\left(t_{i}\right) \equiv L_{A_{i-1} O_{i-1}}\left(t_{i-1}\right)$. Null geodesic of a light signal travelling from a galaxy $\left(A_{1}\right)$ to the observer $O_{i}\left(O_{n} \equiv O\right)$ is also plotted. This picture, of course, wholly agrees with the Cosmological Principle. The requirement for spacial 
homogeneity and isotropy is implemented by this principle in order to avoid a privileged observer. In accord to modern cosmology, the universe does not expand in space, but consists of expanding space. It does not say anything about the point of origin of the universe, either it does not mean that every pair of galaxy $\left(A_{i}\right)$ and observer $\left(O_{i}\right)$ is in any specially favoured or unfavoured position in the universe: the universe is isotropic about this pair, which moving apart as universe expands. Now let us explore the definition of Hubble's parameter to write

$$
H=\frac{d}{d t} \log \left(\frac{R(t)}{R_{1}}\right)=\frac{d}{d t} \ln (1+z)=\frac{1}{1+z} \frac{d z}{d t} .
$$

According to (3), the redshift, $z$ must be expressed in terms of the increment $z=\frac{L-L_{1}}{L_{1}} \equiv \frac{L\left(t_{n}\right)-L\left(t_{1}\right)}{L\left(t_{1}\right)}$, which incorporated with the relations $d t / d t_{1}=1+z$ and (4) yield

$$
\frac{d L_{1}}{d t_{1}}=\dot{L}-H L=0 .
$$

It is then mere question of convenience to think of an observer $\left(O_{(1)}\right)$ having observed in its rest frame of reference that any point of curve $L_{1}\left(=L_{A_{1} O_{(1)}}\right)$ is not receding:

$$
v_{A_{1} O_{(1)}}\left(t_{1}\right)(\text { recession velocity })=0 \quad(\text { redshift }=0) \text {. }
$$

Thus the relation $\lambda_{A_{1}}=\lambda_{O_{(1)}}$ holds for the wavelengths, which is of course consistent with (3). Imagine a family of comoving adjacent observers situated at the points $a_{i}(i=2, \ldots, n)$ on the infinitesimal distances from the galaxies $\left(A_{i}\right)$, who measure the frequency of light rays emitted by $\left(A_{i}\right)$ as it goes by. After making due allowances for (3), particularly, the 'relative' infinitesimal increment $\delta z_{j}$ $(j=1, \ldots, n-1)$ of redshift reads

$$
\delta z_{j}=\frac{\delta \lambda_{j}}{\lambda_{j}}=\frac{\lambda_{j+1}-\lambda_{j}}{\lambda_{j}}=\frac{\delta L_{j}}{L_{j}}=\frac{L_{j+1}-L_{j}}{L_{j}}=\frac{\tilde{\delta} z_{j}}{1+z_{j}} \equiv \frac{z_{j+1}-z_{j}}{1+z_{j}}, \quad 1+z_{j}=\frac{\delta \lambda_{j}}{\lambda_{1}} .
$$

Consequently, an observers should observe the successive increments of 'relative' redshifts, $\delta z_{1}, \delta z_{2}$, $\delta z_{3}, \ldots, \delta z_{n-1}$, of the light when passing across the infinitesimal distances $\left(A_{2}, a_{2}\right),\left(A_{3}, a_{3}\right), \ldots,\left(A_{n}, a_{n}\right)$. Thus, the wavelength of light emitted at $A_{i}$ is infinitesimally stretched out relative to the wavelength of light emitted at the adjacent point $a_{i}$. While weak, such effects considered cumulatively over a great number of successive increments of redshifts could become significant. The resulting redshift is the accumulation of a series of infinitesimal 'relative' redshifts. This interpretation holds rigorously even for large redshifts of order one or more.

If this view would prove to be true, it would lead to the chain rule for the wavelengths:

$$
\frac{\lambda_{A_{n}}}{\lambda_{1}} \equiv \frac{\lambda_{n}}{\lambda_{1}}=\frac{\lambda_{n}}{\lambda_{n-1}} \cdot \frac{\lambda_{n-1}}{\lambda_{n-2}} \cdots \frac{\lambda_{3}}{\lambda_{2}} \cdot \frac{\lambda_{2}}{\lambda_{1}}=\prod_{i=1}^{n-1}\left(1+\delta z_{i}\right),
$$

where $\lambda_{1} \equiv \lambda_{A_{1}}\left(=\lambda_{O_{1}}=\lambda_{O_{n}}\right)$, which readily yields

$$
1+z=\frac{\lambda_{A_{n}}}{\lambda_{O_{n}}}=\prod_{i=1}^{n-1}\left(1+\delta z_{i}\right) .
$$

With no loss of generality, we may of course apply (9) all the way to $n \rightarrow \infty$. Let us view the increment of the proper distance, $L_{i}=L\left(t_{i}\right)$, between a galaxy $\left(A_{i}\right)$ and observer $\left(O_{i}\right)$ over epochs $t_{i} \quad(i=2, \ldots, n)$ as follows: $L_{i}=L_{1}+(i-1) \varepsilon$, where $\varepsilon$ can be made arbitrarily small by increasing $n$. In the limit $n \rightarrow \infty$, all the emitters $\left(A_{i}\right)$ and respective adjacent observers $\left(a_{i}\right)$ are arbitrarily close to each other, the physical separations $\left(A_{i}, a_{i}\right)$ are approaching to zero, so that $\delta z_{i}=\delta L_{i} / L_{i} \simeq \varepsilon / L_{1} \rightarrow 0$. This allows us to write the following relation for the infinitesimal 'relative' redshifts:

$$
\begin{aligned}
\left(\delta z_{n-1}=\delta z_{n-2}\right. & \left.=\cdots=\delta z_{1}=\varepsilon / L_{1}\right)_{n \rightarrow \infty}=\delta z^{(a)}= \\
\lim _{n \rightarrow \infty} \delta z_{(n-1)}^{(a)} & \equiv \lim _{n \rightarrow \infty}\left(\frac{1}{n-1} \sum_{i=1}^{n-1} \delta z_{i}\right),
\end{aligned}
$$

where $\delta z^{(a)}$ is the average infinitesimal increment of 'relative' redshift. The relation (9) then becomes

$$
1+z=\lim _{n \rightarrow \infty} \prod_{i=1}^{n-1}\left(1+\delta z_{i}\right)=\lim _{n \rightarrow \infty}\left(1+\delta z_{(n-1)}^{(a)}\right)^{n-1} .
$$


There does not seem to be any reason to doubt a validity of the relation (10). Certainly, the identification adopted here can be readily proved as follows. According to (7), in curved space of expanding universe, in general, the infinitesimal 'relative' redshifts arise at a series of infinitesimal stretching of the proper distance, so that the relation (11), by virtue of (10), can be recast into the form

$$
1+z=\lim _{n \rightarrow \infty}\left(1+\frac{1}{n-1} \sum_{i=1}^{n-1} \frac{\delta L_{i}}{L_{i}}\right)^{n-1}=\lim _{n \rightarrow \infty}\left(1+\frac{1}{n} \ln \frac{L_{n}}{L_{1}}\right)^{n}=\lim _{n \rightarrow \infty} \frac{L_{n}}{L_{1}},
$$

which agrees with (3). Hence the overall cosmological redshift is a new physical phenomenon consequent on expansion of the universe, which induces the wave stretching of the traveling light via the Lemaître's important relationship:

$$
z=\frac{L(t)}{L\left(t_{1}\right)}-1=\frac{R(t)}{R\left(t_{1}\right)}-1
$$

provided, $(1+z)$ is the factor by which the universe has expanded while the light was travelling towards an observer.

It is worth emphasizing that the general equation (11) is the result of a series of infinitesimal stretching of the proper distance in RW space-time, whereas the path of a light appears nowhere, thus this equation does not relate to the special choice of any transport path. Therefore, to overcome the ambiguity of parallel transport of four-velocities, particularly, in RW space-time, in what follows we advocate exclusively with this proposal. To obtain some feeling about this statement, below we give more detailed explanation.

According to well-known generalization of the spectral shift rule in a Riemannian space-time (Synge, 1960), the infinitesimal increments of 'relative' spectral shifts $\left(\delta z_{1}, \delta z_{2}, \delta z_{3}, \ldots, \delta z_{n-1}\right)$ can be derived from Doppler effect between adjacent emitter and absorber in relative motion measured in the respective tangent local inertial rest frames at infinitesimally separated space-time points. Let $v_{A_{i} a_{i}}\left(t_{i}\right)$ be the relative infinitesimal velocity consequent on recession of a galaxy $\left(A_{i}\right)$ to adjacent observer at the point $a_{i}$, separated by the infinitesimal distance $\delta L_{i} \equiv L_{A_{i} a_{i}}\left(t_{i}\right)$. Since each proper distance $L_{A_{i-1} O_{i-1}}\left(t_{i-1}\right)$ (at epoch $t_{i-1}$ ) is identically mapped on the line segment $L_{a_{i} O_{i}}\left(t_{i}\right)$ of proper distance (at infinitesimally close epoch $t_{i}$ ), the relative velocity $v_{A_{i} a_{i}}\left(t_{i}\right)$ is the same as it is relative to a galaxy $\left(A_{i-1}\right): v_{A_{i} A_{i-1}}\left(t_{i}\right) \equiv v_{A_{i} a_{i}}\left(t_{i}\right)$. Continuing along this line, we may commit ourselves in the series of 'relative' spectral shifts a certain substitution of increments of relative velocities. Taking into account that such infinitesimal relative velocities arise at a series of infinitesimal stretching of the proper distance $L_{A_{2} a_{2}}\left(t_{2}\right), \ldots, L_{A_{n} a_{n}}\left(t_{n}\right)$ as it is seen from the Fig. 1, we may at equal footing fill out now the whole pattern of monotonic increments of 'relative' spectral shifts $\left(\delta z_{1}, \delta z_{2}, \delta z_{3}, \ldots, \delta z_{n-1}\right)$ by replacing the respective pairs $\left(A_{2}, A_{1}\right), \ldots,\left(A_{n}, A_{n-1}\right)$ with new ones $\left(A_{2}, a_{2}\right), \ldots,\left(A_{n}, a_{n}\right)$, which attribute to the successive increments of recession (relative) velocities $v_{A_{2} A_{1}}\left(t_{2}\right), \ldots, v_{A_{n} A_{n-1}}\left(t_{n}\right)$ of a galaxy $\left(A_{n}\right)$ away from observer $\left(O_{n}\right)$ in the rest frame of $\left(O_{n}\right)$, viewed over different epochs $\left(t_{2}, \ldots, t_{n}\right)$. Thereby the principle of time-invariant homogeneity requires that if a galaxy $\left(A_{i}\right)$ recedes from an adjacent observer $\left(a_{i}\right)$ with velocity $v_{A_{i} a_{i}}\left(t_{i}\right)\left(=v_{A_{i} A_{i-1}}\left(t_{i}\right)\right)$, then a galaxy $\left(A_{i-1}\right)$ simultaneously recedes from an equally spaced observer $\left(a_{i-1}\right)$ with the same velocity $v_{A_{i-1} a_{i-1}}\left(t_{i-1}\right)\left(=v_{A_{i-1} A_{i-2}}\left(t_{i-1}\right)\right)$ : $v_{A_{i} a_{i}}\left(t_{i}\right)=v_{A_{i-1} a_{i-1}}\left(t_{i-1}\right)$. This framework furnishes justification for the recession velocity $v_{n} \equiv v_{A_{n} A_{1}}$, to be now referred to as the kinetic recession velocity, of galaxy $\left(A_{n}\right)$ away from observer $\left(O_{n}\right)$, in its rest frame, at epoch $\left(t_{n}\right)$. According to relation (10), at the limit $n \rightarrow \infty$, the infinitesimal recession velocities tend to zero: $v_{A_{i} a_{i}}\left(t_{i}\right)=c \delta \beta_{i}=c \delta z_{i}=c \delta L_{i} / L_{i} \simeq c \varepsilon / L_{1} \rightarrow 0$, such that

$$
\begin{aligned}
& \lim _{n \rightarrow \infty} \delta \beta_{1}=\lim _{n \rightarrow \infty} \delta \beta_{2}=\cdots=\lim _{n \rightarrow \infty} \delta \beta_{n-1}= \\
& \delta \beta^{(a)}\left(\equiv \lim _{n \rightarrow \infty} \frac{1}{n-1} \sum_{i=1}^{n-1} \delta \beta_{i}\right)=\lim _{n \rightarrow \infty} \frac{1}{n} \beta_{n} .
\end{aligned}
$$

Remark: Although we are free to deal with any infinitesimal 'relative' spectral shift $\delta z_{i}$ of emitter $\left(A_{i}\right)$ in local tangent inertial rest frame of adjacent absorber $\left(a_{i}\right)$ (where we may approximate away the curvature of space in the infinitesimally small neighborhood), we should take into account that the infinitesimal relative velocities arise in RW space-time at a series of infinitesimal stretching of the proper distance, and that the SR law of composition of velocities cannot be implemented globally along 
the non-null geodesic, because these velocities are velocities at the different events, which should be in a different physical frames, and cannot be added together.

Facilitating further the calculations of recession velocity $\left(\beta_{n}\right)$ in quest, we may address a galaxy $\left(A_{n}\right)$ and an adjacent observer at $\left(a_{n}\right)$. Suppose $V_{\left(A_{n}\right)}^{\mu}$ and $V_{\left(a_{n}\right)}^{\mu}(\mu=0,1,2,3)$ are the unit tangent four-velocity vectors to their respective world-lines, thus in their respective rest frame we have $V_{\left(A_{n}\right)}^{0}=$ 1 and $V_{\left(a_{n}\right)}^{0}=1$, as the only nonzero components of velocity. Employing a generalization of the spectral shift rule in a Riemannian space-time (Synge, 1960), the infinitesimal increment $\delta z_{n-1}$ of spectral shift can be written

$$
\delta z_{n-1}=\frac{U_{\mu\left(A_{n}\right)} V_{\left(A_{n}\right)}^{\mu}}{U_{\nu\left(a_{n}\right)} V_{\left(a_{n}\right)}^{\nu}}-1,
$$

where $U_{\mu\left(A_{n}\right)}$ and $U_{\mu\left(a_{n}\right)}$ are the tangent vector to null geodesic $\Gamma_{A_{n} a_{n}}$ at end points. The frequency shift $\delta z_{n-1}$ is expressed by the metric tensor, the direction of the velocity of light and the velocities of source and observer. Since all the paths between infinitesimally separated space-time points $\left(A_{n}\right)$ and $\left(a_{n}\right)$ coincide at $n \rightarrow \infty$, there is no need to worry about specific choice of the path of parallel transport of four-vector. Therefore, let us further subject the unit tangent four-velocity vector $V_{\left(A_{n}\right)}^{\mu}$ to parallel transport along the null geodesic joining $\left(A_{n}\right)$ and $\left(a_{n}\right)$. This yields at $\left(a_{n}\right)$ the vector $\beta_{\mu\left(a_{n}\right)}=\mathrm{g}_{\mu \nu^{\prime}}\left(a_{n}, A_{n}\right) V_{\left(A_{n}\right)}^{\nu^{\prime}}$, where the two point tensor $\mathrm{g}_{\mu \nu^{\prime}}\left(a_{n}, A_{n}\right)$ is the parallel propagator, which is determined by the points $A_{n}$ and $a_{n}$. At $\left(A_{n}\right) \rightarrow\left(a_{n}\right)$, we have the coincidence limit $\left[\mathrm{g}_{\mu \nu}\right]\left(a_{n}\right)=$ $g_{\mu \nu}\left(a_{n}\right)$. As we have at point $\left(a_{n}\right)$ two velocities $V_{\left(a_{n}\right)}^{\mu}$ and $\beta_{\left(a_{n}\right)}^{\mu}$, following Synge, (see also Narlikar (1994), we can associate Doppler shift $\delta z_{n-1}$ to a galaxy $\left(A_{n}\right)$ with four-velocity $\beta_{\left(a_{n}\right)}^{\mu}$ observed by an adjacent observer $\left(a_{n}\right)$ with four-velocity $V_{\left(a_{n}\right)}^{\mu}$ as measured by the latter:

$$
\delta z_{n-1}=\frac{U_{\mu\left(a_{n}\right)} \beta_{\left(a_{n}\right)}^{\mu}}{U_{\nu\left(a_{n}\right)} V_{\left(a_{n}\right)}^{\nu}}-1=1-\frac{1}{\left(1+\beta_{\left(a_{n}\right)}^{2}\right)^{\frac{1}{2}}+\beta_{R\left(a_{n}\right)}},
$$

where $c \beta_{\left(a_{n}\right)}^{\mu}=v_{\left(a_{n}\right)}^{\mu}, c \beta_{\left(a_{n}\right)}=v_{\left(a_{n}\right)}, c \beta_{R\left(a_{n}\right)}=v_{R\left(a_{n}\right)}$, and

$$
v_{\left(a_{n}\right)}^{2}=v_{(\alpha)\left(a_{n}\right)} v_{\left(a_{n}\right)}^{(\alpha)}, \quad v_{(\alpha)\left(a_{n}\right)}=v_{\mu\left(a_{n}\right)} \xi_{(\alpha)\left(a_{n}\right)}^{\mu}, \quad v_{R\left(a_{n}\right)}=v_{\mu\left(a_{n}\right)} r_{\left(a_{n}\right)}^{\mu}=v_{(\alpha)\left(a_{n}\right)} v_{\left(a_{n}\right)}^{(\alpha)} .
$$

Reviewing notations the three-velocity of $\left(A_{n}\right)$ relative to $\left(a_{n}\right)$ are defined by the tree invariant components $v_{(\alpha)\left(a_{n}\right)}$, the relative speed is $v_{\left(a_{n}\right)}$, and $v_{R\left(a_{n}\right)}$ is the speed of recession of $\left(A_{n}\right)$. The frame of reference $\xi_{(\alpha)\left(a_{n}\right)}^{\mu}$ defined at $\left(a_{n}\right)$ implies $\xi_{0\left(a_{n}\right)}^{\mu}=V_{\left(a_{n}\right)}^{\mu}$, the unit vector $r^{\mu\left(a_{n}\right)}$ at $\left(a_{n}\right)$ is orthogonal to world-line of $\left(a_{n}\right)\left(r_{\mu\left(a_{n}\right)} V_{\left(a_{n}\right)}^{\mu}=0\right)$ and lying in the 2-element which contains the tangent at $\left(a_{n}\right)$ to world-line of an observer $\left(a_{n}\right)$ and $\left(A_{n}, a_{n}\right)$.

In the local inertial rest frame $\xi_{(\alpha)\left(a_{n}\right)}^{\mu}$ of an observer $\left(a_{n}\right)$, the velocity vector $\beta_{\left(a_{n}\right)}^{\mu}$ takes the form $\left(\gamma, \gamma \delta \beta_{\left(a_{n}\right)}, 00\right)$, where a galaxy $\left(A_{n}\right)$ is moving away from an observer $\left(a_{n}\right)$ with the relative infinitesimal three-velocity $c \delta \beta_{\left(a_{n}\right)}$ in a direction making an angel $\theta_{\left(a_{n}\right)}$ with the outward radial direction from $\left(a_{n}\right)$ to $\left(A_{n}\right)$, and $\gamma=\left(1-\delta \beta_{\left(a_{n}\right)}^{2}\right)^{-1 / 2}$. Hence, the equation (16) is reduced to

$$
\begin{aligned}
& \delta z_{n-1}=\frac{1+\delta \beta_{\left(a_{n}\right)} \cos \theta}{\sqrt{1-\delta \beta^{2}}}-1=\beta_{R\left(a_{n}\right)}-\beta_{R\left(a_{n}\right)}^{2}+\frac{1}{2} \beta_{\left(a_{n}\right)}^{2}+\cdots \simeq \beta_{R\left(a_{n}\right)}= \\
& \frac{p_{(\alpha)\left(a_{n}\right)}^{(\alpha)} v_{\left(a_{n}\right)}^{(\alpha)}}{E_{\left(a_{n}\right)}}=\delta \beta_{\left(a_{n}\right)} \cos \theta_{\left(a_{n}\right)},
\end{aligned}
$$

where $p_{(\alpha)\left(a_{n}\right)}$ and $E_{\left(a_{n}\right)}$ are, respectively, the momentum and energy of light ray as measured locally by an observer $\left(a_{n}\right)$. Thus, at $n \rightarrow \infty$, the wavelength of emitted by a galaxy $\left(A_{n}\right)$ radiation is increased by the first-order Doppler shift caused unambiguously by the infinitesimal recession velocity $\delta \beta_{\left(a_{n}\right)} \equiv \delta \beta_{n-1}^{(r)}$ in radial direction $\left(\cos \theta_{\left(a_{n}\right)} \rightarrow 1\right)$ :

$$
\delta z_{n-1}=\frac{\delta L_{n-1}}{L_{n-1}}=\delta \beta_{n-1}^{(r)} .
$$


In the local tangent inertial rest frame of an observer $\left(a_{n}\right)$, the latter reads (see subsect. 3.1):

$$
\delta \beta_{n-1}^{(r)}=\frac{\beta_{n}-\beta_{n-1}}{1-\beta_{n} \beta_{n-1}} \simeq \frac{\delta \beta_{n-1}}{1-\beta_{n-1}^{2}},
$$

where $v_{n}=c \beta_{n} \equiv c V_{\left(A_{n}\right)}$ and $v_{n-1}=c \beta_{n-1} \equiv c V_{\left(a_{n}\right)}$ are, respectively, the three-velocities of a galaxy $\left(A_{n}\right)$ and an observer $\left(a_{n}\right)$ along the radial direction from $\left(a_{n}\right)$ to $\left(A_{n}\right)$. A resulting infinitesimal increment $\delta z_{n-1}$ of spectral shift, at $n \rightarrow \infty$ then reads

$$
\lim _{n \rightarrow \infty} \delta z_{n-1}=\lim _{n \rightarrow \infty} \frac{\delta \beta_{n-1}}{1-\beta_{n-1}^{2}}=\lim _{n \rightarrow \infty} \frac{\beta_{n}}{n\left(1-\beta_{n}^{2}\right)},
$$

For our goal, the most straightforward guess at the convenient form of (13), by virtue of (10), is written

$$
1+z=\frac{R(t)}{R\left(t_{1}\right)}=\lim _{n \rightarrow \infty}\left(1+\delta z_{n-1}\right)^{n} .
$$

Certainly, it is merely the choice to be rewarding to go ahead with a finite spectral shift

$$
1+z=\frac{R(t)}{R\left(t_{1}\right)}=\lim _{n \rightarrow \infty}\left[1+\frac{1}{n}\left(\frac{\beta_{n}}{\left(1-\beta_{n}^{2}\right)}\right)\right]^{n} .
$$

This straightforwardly yields the general kinematic relationship of the overall cosmological redshift, $z$, and kinetic recession velocity $\beta_{\text {rec }}$ (in units of the speed of light) of the comoving distant galaxy $\left(A_{1}\right)$ of redshift $z$, which crossed past light cone at time $t_{1}$ away from comoving observer $(O)$ :

$$
1+z=\frac{R(t)}{R\left(t_{1}\right)}=\exp \left(\frac{\beta_{r e c}}{1-\beta_{r e c}^{2}}\right),
$$

where, hereinafter, the kinetic recession velocity $\beta_{n}$ is marked with subscript ()$_{\text {rec }}$. This interpretation so achieved has physical significance as it agrees with a view that the light waves will be stretched by travelling through the expanding universe, and in the same time the kinetic recession velocity of a distant astronomical object is always subluminal even for large redshifts of order one or more. It, therefore, does not violate the fundamental physical principle of causality.

The kinetic recession radial velocity of a galaxy is plotted on the Fig. 2 (Top panel(a): for redshifts $0 \leq z \leq 10$; and Bottom panel(b): for redshifts $0 \leq z \leq 800$ ), where the global Doppler velocity, and their difference are also presented to guide the eye. As it can be seen from the Figure 2, the difference of global Doppler velocity and kinetic recession velocity, for redshifts $0.9 \leq z \leq 800$, is $\left(\beta_{\text {Dop }}-\beta_{\text {rec }}\right) \geq 0.072 c$, where a maximum value, $\left(\beta_{\text {Dop }}-\beta_{\text {rec }}\right)_{\max }=0.187 c$, is reached at redshifts $=4.5-5.1$.

If, and only if, for the distances at which the Hubble empirical linear 'redshift-distance' law $(c z=H L)$ is valid, the relationship between the physical recession velocity, $v_{r e c}$, and the expansion rate, $\dot{L}(=H L)$, reads

$$
\beta_{\text {rec }}=\frac{\sqrt{1+4 \ln ^{2}(1+\dot{L} / c)}-1}{2 \ln (1+\dot{L} / c)} .
$$

Once we are equipped with the general solution, it is worth emphasizing the importance of the parameter $\zeta(z)$ of practical measure of being swept up of galaxy in expanding universe:

$$
\zeta(z)=\frac{v_{r e c}}{\dot{L}}=\frac{\beta_{r e c}}{z} .
$$

Next we will study a particular case of establishing a global Doppler shift from a general solution (24).

\subsection{A global Doppler shift along the null geodesic}

Once we are equipped with the general solution (24), it is worth to show that this solution is reduced to a global Doppler shift along the null geodesic, previously studied by Synge (1960) (see also Bunn \& Hogg (2009), Narlikar (1994)) who used the parallel transport of source four-velocity along the null geodesic to an observer. Certainly, suppose a dense family of adjacent comoving observers being in free fall populated along the path of light ray from a galaxy $\left(A_{1}\right)$ to an observer $\left(O_{n}\right)$ (Fig. 1). The $(i)$-th 

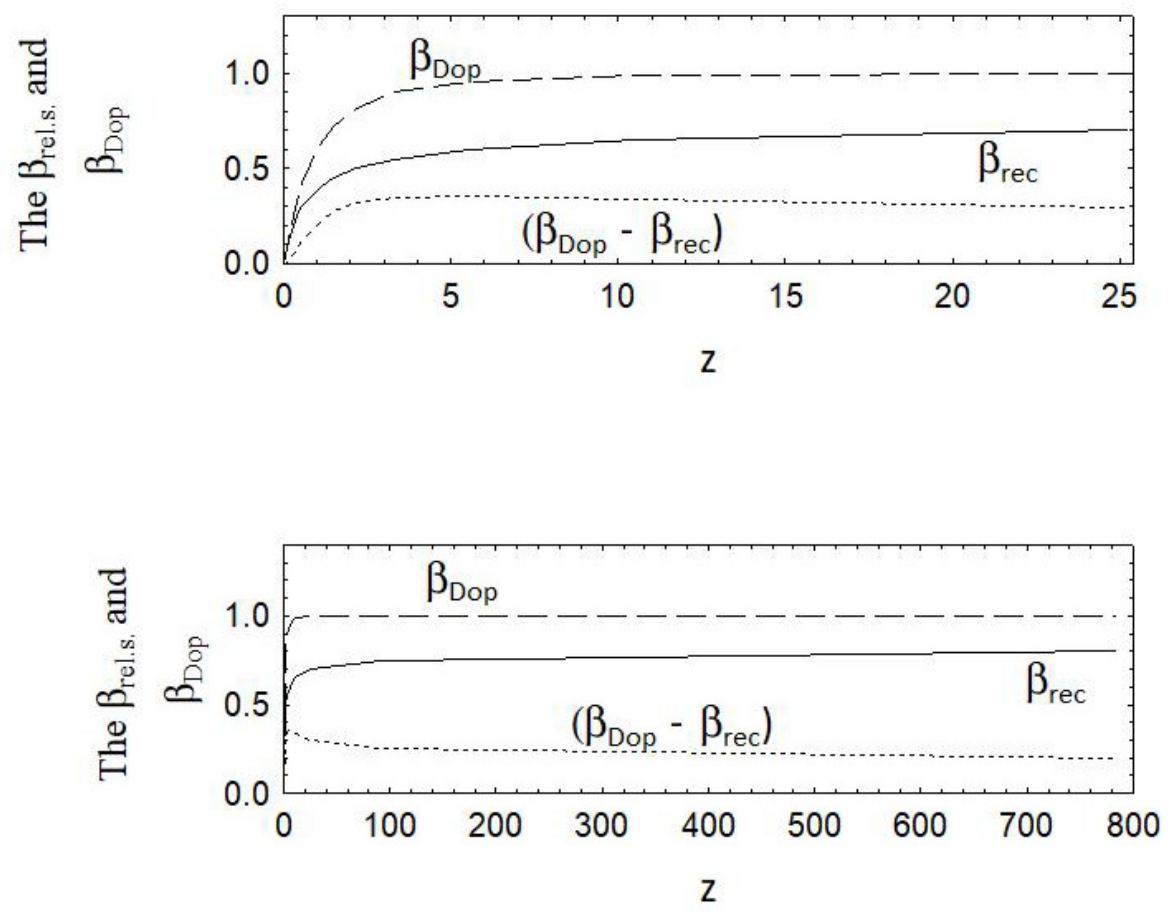

Figure 2. The recession velocity along the line of sight $\left(\beta_{\text {rec }}\right)$ of luminous source $(S)$ with redshift $z$ away from the observer $(O)$, the global Doppler velocity $\left(\beta_{D o p}\right)$, and their difference (in units of the speed of light). Top panel: $0 \leq z \leq 25$; Bottom panel: $0 \leq z \leq 800$.

observer situated at the point $(i)$ of intersection of the ray's trajectory with a comoving hypersurface $S\left(t_{i}\right)$ of constant cosmic time. Then, the end points of infinitesimal distance $\delta l_{i}=c \delta t_{i}$ between the adjacent observers $(i+1)$ and $(i)$ will respectively be the points of intersection of the ray's trajectory with the comoving hypersurfaces $S\left(t_{i+1}\right)$ and $S\left(t_{i}\right)$. Thus the infinitesimal increment of the frequency shift on the distance $\delta l_{i}=c \delta t_{i}$, caused by expansion of the universe during the infinitesimal epoch time interval $\delta t_{i}=t_{i+1}-t_{i}$, according to (7), should be $\delta z_{i}=\delta \lambda_{i} / \lambda_{i}=\delta L_{i} / L_{i}$. Due to the equivalence principle, we may approximate away the curvature of space in the infinitesimally small neighborhood of two adjacent observers. Thereby, approximating away the curvature of spacetime in the infinitesimally small neighborhood does not mean approximating away the expansion altogether. That is, it must be stated emphatically that if we approximate an infinitesimally small neighborhood of the size $\delta L_{i}$ of an expanding spacetime as flat, the resulting errors are of order $\left(\delta L_{i} / L_{H}\right)^{2}$ in the metric. If we regard such errors as negligible, then we can legitimately approximate spacetime as flat. The increment of redshift $\delta z_{i}$ is not approximated away in this limit because it is in that neighborhood of leading order $\left(\delta L_{i} / L_{i}\right)$. Imagine a thin world tube around the null geodesic within which the space-time is flat to arbitrary precision. In particular, this implies the vacuum value of a velocity of light to be universal maximum attainable velocity of a material body found in this space. This statement is true for any thin neighborhood around a null geodesic. Therefore, each observer has a local reference frame in which SR can be taken to apply, and an observers are close enough together that each one $(i+1)$ lies within the local frame of his neighbor $(i)$. Only in this particular case of null geodesic, the relative velocity of observers can be calculated by the SR law of composition of velocities globally along this path. Within each local inertial frame, there are no gravitational effects, and hence the infinitesimal frequency shift from each observer to the next is a Doppler shift. Hence, at the limit $n \rightarrow \infty$, a resulting infinitesimal frequency shift $\delta z_{i}$, can be unambiguously equated to infinitesimal increment of a fractional SR Doppler shift $\delta \bar{z}_{i}$ from observer $(i+1)$ to the next $(i)$ caused by infinitesimal relative velocity $\delta \bar{\beta}_{i}^{r}$ :

$$
\left(\delta z_{i}=\frac{\delta L_{i}}{L_{i}}\right)_{n \rightarrow \infty}=\left(\delta \bar{z}_{i}=\delta \bar{\beta}_{i}^{r}=\frac{\bar{\beta}_{i+1}-\bar{\beta}_{i}}{1-\bar{\beta}_{i+1} \bar{\beta}_{i}} \simeq \frac{\delta \bar{\beta}_{i}}{1-\bar{\beta}_{i}^{2}}\right)_{n \rightarrow \infty},
$$


where by $\overline{(})$ we denote the null-geodesic value, as different choice of geodesics yields different results for the motion of galaxy relative to a particular observer. The relation (27), incorporated with the identity (10), yield

$$
\left(\delta z_{n-1}=\right)_{n \rightarrow \infty}=\left(\frac{\delta \beta_{n-1}}{1-\beta_{n}^{2}}\right)_{n \rightarrow \infty}=\left(\delta \bar{z}_{(n-1)}^{(a)}=\delta \bar{\beta}_{(n-1)}^{r(a)} \equiv \frac{1}{n-1} \sum_{i=1}^{n-1} \frac{\delta \bar{\beta}_{i}}{1-\bar{\beta}_{i}^{2}}\right)_{n \rightarrow \infty},
$$

which, by virtue of (14), for sufficiently large but finite $n$ gives

$$
\frac{\beta_{n}}{1-\beta_{n}^{2}}=\sum_{i=1}^{n-1} \frac{\delta \bar{\beta}_{i}}{1-\beta_{i}^{2}}=\int_{0}^{\bar{\beta}_{n}} \frac{d \bar{\beta}}{1-\beta^{2}},
$$

or

$$
\bar{\beta}_{n}=\frac{e^{\varrho_{n}}-1}{e^{\varrho n}+1}, \quad \varrho_{n} \equiv \frac{2 \beta_{n}}{1-\beta_{n}^{2}} .
$$

Hence the general solution (24), by means of (29), is reduced to a global Doppler shift along the null geodesic:

$$
1+z=\exp \left(\frac{\beta_{r e c}}{1-\beta_{r e c}^{2}}\right)=\sqrt{\frac{1+\bar{\beta}_{r e c}}{1-\bar{\beta}_{r e c}}}
$$

where $\bar{\beta}_{\text {rec }}=\lim _{n \rightarrow \infty} \bar{\beta}_{n}$. Thus, this procedure in fact is exactly equivalent to performing parallel transport of the source four-velocity in curved space of expanding universe along the null geodesic to an observer. In Minkowski space a parallel transport of vectors is trivial and mostly not mentioned at all. This allows us to apply globally the SR law of composition of velocities to relate the velocities $\bar{\beta}_{i+1}$ to the $\bar{\beta}_{i}$ of adjacent observers along the path of light ray, measured in the $i$-th adjacent observer's frame. Then, according to (27)-(31), a global Doppler shift of light ray emitted by luminous source as it appears to observer at rest in flat Minkowski space can be derived by summing up the infinitesimal Doppler shifts caused by infinitesimal relative velocities of adjacent observers.

\section{A cosmological toy model of FRW-universe for zero-density in the RW coordinates}

In our actual universe space-time is not exactly flat, but a sufficiently large region of the transparent universe, say on length scales of one to ten or so billion light years, usually can be well approximated by a zero-density spatially flat homogeneous isotropic cosmological model having $(k=0)$ FRW metric (Page 2009):

$$
d s^{2}=c^{2} d t^{2}-R^{2}(t) d \chi^{2}
$$

where $d \chi=\sqrt{d x^{2}+d y^{2}+d z^{2}}$ is the (constant) comoving coordinate distance between the comoving two particles, with $(d x, d y, d z)$ being the differences between their comoving coordinates. The particles in this idealized model each stay at fixed comoving coordinates $(x, y, z)$ as their proper time $t$ increases. The physical distance between two particles, as measured along a geodesic of a comoving hypersurface of constant $t$, grows at the Hubble expansion rate of the universe at the time $t$. For the spatially flat model (32), there is no upper limit to the comoving coordinate distance $\chi$, and that also to the proper distance $L(t)=R(t) \chi$, at any fixed $t$. The metric (32) can be rewritten as an expanding open RW-metric $(k=-1)$ with $R(t) \propto t$ (Gron 2007; Page 2009), i.e. the Milne model (Milne 1934). The Milne universe is the Minkowski space-time described from an expanding reference frame. Although in the RW coordinates the 3D comoving hypersurface of constant $t$ (constant proper time) does have an extrinsically non-zero curvature, nevertheless the $4 \mathrm{D}$ curvature is zero. Therefore, simple coordinate transformations transform the metric to the standard Minkowski form with the Minkowski coordinates. The Minkowski coordinates $(T, X, Y, Z)$ are the coordinates of a rigid inertial reference frame of an arbitrarily chosen reference particle $P$ in the expanding cloud of particles defining the Milne universe model. The time $T$ is the private time of $P$. The time $t$ is measured on clocks following all of the reference particles. The Milne universe can be identified as the forward light cone in Minkowski space-time, foliated by negatively curved hyperboloids orthogonal to the time axis. In the inertial and rigid Minkowski coordinate system the velocity of a reference particle with comoving coordinate $\chi$ is less than the speed of light for all values of $\chi$. The components of a parallel transported four-vector in 
inertial coordinates are constant and, thus, a parallel transport is trivial and mostly not mentioned at all. As mentioned in Subsection 3.1, the general equation (11) of redshift is unambiguously reduced in this limit to a conventional global Doppler shift formula with SR-relative speed, because the SR law of composition of velocities can be implemented globally in the whole Minkowski space. Thus, in this limit a correspondence to the more usual notion of SR-relative speed retains.

But this is no longer true for non-inertial coordinates RW of expanding cosmic frame. In this frame, the reference particles with Milne coordinates $(t, \chi)$ define the expanding public space of the universe model. The Hubble's expansion refers to the GR space defined by simultaneity on the clocks following the reference particles. It is valid in the public space of the universe model, not the private space of a particular observer. It has infinite extension. Hence the reference particles have superluminal velocity at sufficiently great distances from an observer. Moreover, in the extrinsically curved 3D comoving hypersurface $t=$ const (public space) a parallel transport is not trivial and needs consideration (Page 2009). A geodesic between two events on such hypersurface passes through the future, and a transport along this geodesic will yield a different result. Using the length of geodesics of a particular spatial hypersurface worsens the problem of superluminal expansion. The infinitesimal relative velocities arise at a series of infinitesimal stretching of the proper distance, and that the SR law of composition of velocities cannot be implemented globally in the curved 3D hypersurface. In this case, the relation (11) leads to the general solution (24) of the overall cosmological redshift, with a kinetic recession velocity, which is always subluminal even for large redshifts of order one or more.

\section{Concluding remarks}

The conceptual and technical problems involved in this contribution provide scope for the arguments discussed, aiming to reconcile the cosmological interpretation of redshift with the most natural kinematic interpretation. Below we briefly reflect upon a few relevant points. The solutions given in present report demonstrate its advantage over the specific extant definitions in the cited literature:

- Section 3 presents what is essentially a correct solution to a kinematic interpretation. In the framework of "stretching of space" point of view of the spatially homogeneous and isotropic RW space-time of standard cosmological model, we overcome an ambiguity of the procedure of parallel transport of source four-velocity along the null geodesic to an observer by an alternative study of a "lookforward" history of expanding universe. We use a way of separating the cosmological redshifts into infinitesimal 'relative' redshift bins and sum over them to achieve an unique definition of the kinetic recession velocity of comoving astronomical object. The latter is always subluminal even for large redshifts of order one or more, so that it does not violate the fundamental physical principle of causality. The difference of global Doppler velocity and kinetic recession velocity, for redshifts $0.9 \leq z \leq 800$ is $\geq 0.072 c$, where a maximum value, $0.187 c$, is reached at redshifts $z=4.5-5.1$. We calculate a practical measure parameter $\zeta(z)$ of being swept up of galaxy in expanding universe. We show that the derived general solution is unambiguously reduced to global Doppler shift along null geodesic.

- We discuss the obtained results in the limit of a zero-density cosmological model of Milne universe. Whereas, in the inertial and rigid Minkowski coordinate system the components of a transported four-vector in inertial coordinates are constant and, thus, a parallel transport is trivial and mostly not mentioned at all. The general solution (24) of redshift is unambiguously reduced in this limit to a conventional global Doppler shift with SR-relative speed, because the SR law of composition of velocities can be implemented globally in the whole Minkowski space. Thus, in this limit a correspondence to the more usual notion of SR-relative speed retains. But this is no longer true for non-inertial RW coordinates of expanding cosmic frame. In this frame, the reference particles with Milne coordinates define the expanding public space of the universe model. In the extrinsically curved 3D hypersurface (public space) a parallel transport is not trivial and needs consideration. The infinitesimal relative velocities arise at a series of infinitesimal stretching of the proper distance so that the SR law of composition of velocities cannot 
be implemented globally in the curved 3D hypersurface. In this case, the overall cosmological redshift is described by the formula (24) with a kinetic recession velocity.

- In Appendix A, we give a reappraisal in a deep way of the preliminary attempts of 'standard' kinematic interpretation of redshifts as accumulated Doppler-shifts consequent on recession, widely discussed in literature. Its study is valuable as affording insight into the whole subject. In doing this, we are not suggesting any doubt about the principle statement. Rather, we doubt the method of calculations, which as we have shown are in error. Moreover, the principle statement is a crux of our derivation of an essentially correct solution to a kinematic interpretation of cosmological redshifts. In appendix $\mathrm{B}$, we calculate the kinetic recession velocities of some typical distant astronomical objects with spectroscopic redshift determinations collected from the literature, which are listed in Table 1.

\section{References}

Bahcall J. N., Oke J. B., 1971, ApJ, 163, 235

Bañados E., Venemans B., Morganson E. e. a., 2014, AJ, 148, 14

Bañados E., Venemans B. P., Mazzucchelli C. e. a., 2018, Nature, 553, 473

Bolós V. J., 2006, J. Geom. Phys., 56, 813

Bolós V. J., 2007, Commun. Math. Phys., 273, 217

Bolós V. J., Klein D., 2012, Gen Relativ Gravit., 44, 1361

Bolós V. J., Liern V., Olivert J., 2002, Int. J. Theor. Phys., 41, 1007

Bunn E. F., Hogg D. W., 2009, Am. J. Phys., 77, 6888

Cattaneo C., 1958, Nuovo Cimento, 2, 318

Chambers R. H., 2011, BAAS, 43, 217

Chodorowski M. J., 2011, MNRAS, 413, 585

Davis T. M., Lineweaver C. H., 2004, PASAu, 21, 97

Dirac P. A. M., 1979, Proc. R. Soc. London A, 365, 19

Eckart C., 1940, Pys.Rev., 58, 919

Edge A e. a., 2013, Msngr, 154, 32

Ellis G. F. R. e. a., 1985, Phys. Rep., 124, 315

Emtsova E., Toporensky A., 2019, arXiv:[gr-qc], 1901.03602, 1

Fan X., Strauss M. A., Schneider D. P. e. a., 1999, AJ, 118, 1

Fan X., Strauss M. A., Richards G. T. e. a., 2001, AJ, 121, 31

Fan X., Carilli C. L., Keating B., 2006a, ARA\&A, 44, 415

Fan X., Strauss M. A., Richards G. T. e. a., 2006b, AJ, 131, 1203

Fan X., Strauss M. A., Becker R. H. e. a., 2006c, AJ, 132, 117

Gautreau R., 1984, Phys. Rev. D, 29, 198

Grøn Ø., Elgarøy Ø., 2007, Am. J. Phys., 75, 151

Grøn Ø., Rippis P. D., 2003, Gen. Re. Grav., 35, 2189

Harrison E. R., 1993, ApJ, 403, 28

Harrison E. R., 1995, ApJ, 446, 63

Harrison E. R., 1981 and 2000, Cosmology: the science of the universe, 1 and 2 edn. Cambridge U. Press, Cambridge

Hashimoto T., Laporte N., Mawatari K. e. a., 2018, Nature, 557, 312

Hu Y., Turner M. S., Weinberg E. J., 1993, Phys. Rev. D, 49, 3830 
Hu E. M., Cowie L. L., McMahon R. G. e. a., 2002, ApJ, 568, L75

Iwamuro F., Motohara K. e. a., 2002, ApJ, 565, 63

Jiang L., Fan X., Vestergaard M. e. a., 2007, AJ, 134, 1150

Jiang L., Fan X., Annis J. e. a., 2008, AJ, 135, 1057

Jiang L., McGreer I. D., Fan X. e. a., 2016, ApJ, 833, 222

Kaiser N., Aussel H., Burke B. E. e. a., 2002, in Society of Photo-Optical Instrumentation Engineers (SPIE) Conference Series. p. 154

Kaiser N., Burgett W., Chambers K. e. a., 2010, in Society of Photo-Optical Instrumentation Engineers (SPIE) Conference Series. p. $77330 \mathrm{E}$

Kashikawa N. e. a., 2015, ApJ, 798, 28

Kaya A., 2011, Am. J. Phys., 79, 1151

Klein D., Collas P., 2010, Phys. Rev. D, 81, 063518

Klein D., Randles E., 2011, Ann. Henri Poincaré, 12, 303

Kurk J. D., Walter F., Fan X. e. a., 2009, ApJ, 702, 833

Laporte N., Ellis R. S., Boone F. e. a., 2017, ApJ, 837, L21

Lawrence A., 2007, MNRAS, 379, 1599

Leaf B., 1951, Pys.Rev., 84, 345

Liebscher D.-E., 2007, Astron. Nachr. / AN, 328, 586

Lilly S. J., Longair M. S., 1984, MNRAS, 211, 833

Lineweaver C. H., Davis T. M., 2005, SciAm, 292, 36

Lynds R., Wills D., 1970, Nature, 226, 532

Manasse F. K., Misner C. W., 1963, J. Math. Phys., 4, 735

Massa E., 1974, Gen.Rel. and Grav., 5, 555, 573, 715

Matsuoka Y. e. a., 2016, ApJ, 828, 26

McConnell N. J., Ma C.-P., Gebhardt K. e. a., 2011, Nature, 480, 215

McVittie C. C., 1933, MNRAS, 93, 325

Milne E. A., 1934, Quarterly J. of Math., 5, 64

Mortlock D. J., Warren S. J., Venemans B. P. e. a., 2011, Nature, 474, 616

Murdoch H. S., 1977, QJRAS, 18, 242

Narlikar J. V., 1994, Am. J. Phys., 62, 903

Oesch P. A., Brammer G., van Dokkum P. G. e. a., 2016, ApJ, 819, 129

Padmanabhan T., 1993, Structure Formation in the Universe. Cambridge Univ. Press, Cambridge

Page D. N., 2009, Class. Quantum Grav., 26, 127001

Pâris I., Petitjean P., Aubourg É. e. a., 2012, A\&A, 548A, 66

Pâris I., Petitjean P., Aubourg É. e. a., 2014, A\&A, 563A, 54

Peacock J. A., 1999, Cosmological Physics. Cambridge Univ. Press (Ninth printing with corrections 2010), address $=$ Paris

Peacock J. A., 2002, in Modern Cosmology, Proceedings of the Como School 2000, ed. by Bonometto, S and Gorini, V and Moschella U. IOP, Bristol

Peacock J. A., 2008, arXiv:[astro-ph], 0809.4573, 1

Peebles P. J. E., 1993, Principles of Physical Cosmology. Princeton Univ.Press.

Peebles P. J. E., Schramm D., Turner E., Kron R., 1991, Nature, 352, 769

Pirani F., 1962, Le Theories relativistes dee la Gravitation. CNRS, Paris 
Pössel M., 2019, arXiv: [gr-qc], 1912.11677, 1

Postman M., Lauer T. R., Donahue M. e. a., 2012, ApJ, 756, 159

Reed S. L., McMahon R. G., Banerji M. e. a., 2015, MNRAS, 454, 3952

Robertson H. P., 1935, ApJ, 82, 284

Scharẅachter J., Combes F., Salomé P., Sun M., Krips M., 2016, MNRAS, 457, 4272

Schmidt M., Schneider D. P., Gunn J. E., 1987, ApJ, 321, L7

Schneider D. P., Richards G. T., Hall P. B. e. a., 2010, AJ, 139, 2360

Shanks T. e. a., 2015, MNRAS, 451, 4238

Silverman A. N., 1986, Am. J. Phys., 54, 1092

Stern D., Spinrad H., 1999, PASP, 111, 1475

Stern D., Moran E. C., Coil A. L. e. a., 2002, ApJ, 568, 71

Stuckey W. M., 1992, Am. J. Phys., 60, 142

Synge J. L., 1960, Relativity: The General Theory. North-Hollaand, Amsterdam

Tanvir N. R., Fox D. B., Levan A. J. e. a., 2009, Nature, 461, 1254

Thomas J., Ma C.-P., McConnell N. J., Greene J. E., Blakeslee J. P., Janish R., 2016, Nature, 532, 340

Turner E., 2001, AJ, 101, 5

Vanzella E., Pentericci L., Fontana A. e. a., 2011, ApJ Lett., 730, L35

Venemans B. P., Findlay J. R., Sutherland W. J. e. a., 2013, ApJ, 779, 24

Vladimirov I. S., 1982, Reference systems in gravitation theory. Energoizdat, (In Russian), Moscow

Walker A. G., 1935, Proc. Lond. Math. Soc., 4, 170

Walker A. G., 1936, Proc. Lond. Math. Soc., 42, 90

Wang R., Carilli C. L., Beelen A. e. a., 2007, ApJ, 134, 619

Wang F., Wu X. B., Fan X. e. a., 2016, ApJ, 819, 24

Warren S. J., Hewett P. C., 1990, Rep. on Prog. in Phys., 53, 1095

Warren S. J., Hewett P. C., Osmer P. S. e. a., 1987, Nature, 330, 453

Watson D., Christensen L., Knudsen K. K. e. a., 2015, Nature, 519, 327

Whiting A. B., 2004, The Observatory, 124, 174

Willott C. J., Delorme P., Omont A. e. a., 2007, AJ, 134, 2435

Willott C. J., Albert L., Arzoumanian D. e. a., 2010, AJ, 140, 546

Wolf C., Onken C. A., Luvaul L. C. e. a., 2018, PASA, 35, 10

Wu X.-B., Wang F., Fan X. e. a., 2015, Nature, 518, 512

Yang J., Fan X., Wu X.-B. e. a., 2017, AJ, 153, 184

Zelmanov A. L., 1944, Chronometric Invariants. Am. Research Press (Tr. 2006), New Mexico, USA

Zelmanov A. L., 1956, Dokl. Acad. Nauk USSR, 107, 815

Zelmanov A. L., 1976, Dokl. Acad. Nauk USSR, 227, 78

Zitrin A., Labbé I., Belli S. e. a., 2015, ApJ, 810, L12 


\section{Appendices}

\section{Appendix A The standard kinematic interpretation of redshifts}

One source of the preliminary attempts along this line of alternative understanding of a complex problem is the "standard" kinematic interpretation that cosmological redshift of distant galaxy is the recession effect of the accumulation of a series of infinitesimal Doppler shifts due to infinitesimal relative velocities of the Hubble flow along the line of sight. Following Padmanabhan (1993), Peacock (1999, 2008), Peebles (1993), (see also e.g. Bunn \& Hogg (2009), Chodorowski (2011), Grøn \& Elgarøy (2007), Whiting (2004)), within the "stretching of space" point of view, assume that an observer at the origin at the present epoch time measures the redshift of a galaxy at some comoving distance. Consider a light ray that travels from a galaxy to this observer, both of whom are at rest in comoving coordinates. Imagine a family of comoving observers along the path of light ray, each of whom measures the frequency of light ray as it goes by. It was assumed that each observer is close enough to his neighbor so that we can accommodate them both in one inertial reference frame and use SR to calculate the change in frequency from one observer to the next. If adjacent observers are separated by the infinitesimal proper distance $\delta L$, then their relative velocity in this frame is $\delta v=H \delta L$. This infinitesimal recessional velocity should cause a fractional shift given by the non-relativistic Doppler formula:

$$
\frac{\delta \nu}{\nu}=-\frac{\delta v}{c}=-\frac{H \delta L}{c}=-H \delta t .
$$

And hence, as it was concluded, the relation (33) for overall redshift, by means of $H=\dot{R} / R$, becomes $\frac{\delta \nu}{\nu}=-\frac{\delta R}{R}$. This integrates to give the main result of expansion scenario that the frequency decreases in inverse proportion to the scale factor, $\nu \propto 1 / R$.

It was claimed even more that the cosmological redshifts can be interpreted as a combination of Doppler and gravitational shifts with the difference in gravitational potential between the point of emission and reception of a light ray. It is the purpose of this Section to give a reappraisal of the key aspects of the "standard" interpretation in a deep way. We wish to make clear at the outset that we are not suggesting any doubt about the principle statement that the redshift of distant galaxy is the recession effect of the accumulation of a series of infinitesimal Doppler shifts due to infinitesimal relative velocities along the line of sight, which seemed appealing and attractive. Moreover, this statement has been a crux of our derivation of a correct solution to a kinematic interpretation of cosmological redshifts presented in Section 4. Rather, we doubt the method of calculations. A hard look at the basic relation (33) reveals the following objections, which together constitute a whole against the claim:

- Even this worry is subtle, but here we have called attention to the fact that there is no necessity for integration of (33) to reveal the "stretching-of-wavelength" effect, because the term of assumed expansion dynamics, $H \delta L$, written for the infinitesimal change of expansion rate on the infinitesimal distances, $\delta L$, is already steeped in (33), which implies the wave-stretching relation, $\delta \lambda \propto \delta L \propto \delta R$.

- We doubt a validity of a scale-behavior $\nu \propto 1 / R$ of the frequency that enters the equation (33). Upon closer examination, the arguments about this are completely futile because they are conducted without any attempt to analyze the meanings of the terms employed in (33). The factor $1+z$ measures the expansion of the universe between emission and absorption of a light. The equation (33) would lead to the relation $\frac{\delta \nu}{\nu}=-\frac{\delta R}{R}$ if, and only if, $\dot{L}(t, z)=c$, i.e. when galaxy situates on the Hubble sphere: $L=L_{H} \equiv c / H$, where $L_{H}$ is the Hubble length. But in general case of $\dot{L}(t, z) \neq c\left(L \neq L_{H}\right)$, it was in conflict because the infinitesimal time interval $\delta t^{\prime}=\delta L / c$ does not equal to the infinitesimal epoch time interval $\delta t=\delta L / \dot{L}\left(\delta t^{\prime} / \delta t=\dot{L} / c\right)$ :

$$
\frac{H \delta L}{c}=\frac{1}{R}\left(\frac{d R}{d t} \delta t\right) \frac{\delta L}{c \delta t}=\frac{\dot{L}(t, z)}{c} \frac{\delta R}{R},
$$

and hence (33) leads to

$$
\frac{\delta \nu}{\nu}=-\frac{\dot{L}(t, z)}{c} \frac{\delta R}{R} .
$$


This can readily be transformed into the equation

$$
\delta \ln \nu=\delta\left[\ln \left(\frac{1}{R}\right)^{\dot{L} / c}\right]+\frac{\delta \dot{L}}{c} \ln R .
$$

As it is evidently seen, the result of integration of (36) will be utterly distinct from the simple behavior of $\nu \propto 1 / R$.

- To render our discussion more transparent, it is worthwhile to clarify the ingredient relation $\delta v=H \delta L$ in (33), where $\delta v$ is the Doppler velocity: $c \delta z=\delta v$. We argue that this relation is in error at the infinitesimal distances. It is necessary to substantiate this principle statement further by the reasoning recast in more physical terms. For the self-contained arguments, it must be stated emphatically that the Hubble's linear 'redshift-distance' law, $c z=H L$, certainly must be classified as an empirical law rather than as a law of pure reason. It must then suffice to expect some objections against its idealization and, thus, it should be a subject of limited validity (an approximate relation). One ought to be cautious about a validity of this law in the vicinity of nearby clusters of galaxies, or on much smaller scales. It seems there is rather no local counterpart for the dynamics from the Hubble law on the much smaller distances, say in the vicinity of a star such as our Sun. In this, the Schwarzschild field will dominate, with the cosmological field perhaps exerting some small perturbative effects (Dirac, 1979, Gautreau, 1984, Grøn \& Rippis, 2003, McVittie, 1933). Needless thus to say that the Hubble's linear "redshiftdistance" law becomes a dangerously flawed way of thinking and cannot be representative of the global behavior at least on the infinitesimally smaller distances, because if this law could affect local dynamics this should contradict profoundly to principle of "relativity". Our conclusion drawn from the above discussion can be stated as follows:

$$
\delta v=c \delta z \neq H \delta L
$$

- We further argue that the cosmological redshift, in general, cannot be interpreted as a combination of the global Doppler and gravitational shifts. Certainly, Narlikar (1994) has presented the proof that as the universe expands, the parallel transported in curved space four-velocity vector of a distant source along the null geodesic to an observer does yield Doppler four-velocity in the rest frame of observer. This result is particular case of a general rule described in comprehensible terms by Synge (1960) (Chap.III). We seem to have attractive proposal of choosing a null geodesics for the parallel transport since it does not require any additional structures, like particular foliation of space-time, which in turn is applicable to any space-time. However, a resulting global Doppler effect is inconclusive, and could not be regarded as a final word, because a definition of relative velocity has disadvantage that in a curved space-time for Levi-Civita connection the result of parallel transport depends on the chosen path along which the vector is transported. This is the defining property of curvature. Hence, there is no relative velocity of distant objects without prior choice of transport paths. If such definition of relative velocity is not accepted, then the statement attributing frequency shifts to relative velocity cannot be accepted either.

- Finally, recall that the gravitational redshift occurs if light travels from a strong to a weak gravitational field and blueshift occurs for the light traveling in the reverse direction. But the cosmological redshift occurs in both these cases as the universe expands. Moreover, the GR interpretation of the expansion interprets cosmological redshift as an indication of velocity since the proper distance between comoving objects increases. Since the Riemann tensor appears nowhere, thus the cosmological redshifts do not relate to gravitational effect. 


\section{Appendix B The kinetic recession velocities of some typical distant astronomical objects}

A tremendous observational effort has been put in many decades into the programme of detection and characterization of distant galaxies and high redshift bright quasars, which is pivotal for elucidating the physics in time to an epoch when the universe was very young and galaxies in their infancy. The high redshift bright quasars have fundamental implications for the formation and growth history of supermassive black holes ((SMBHs), see e.g. (Mortlock et al., 2011, Wu et al., 2015), and probe the progress of cosmic reionisation (Fan et al., 2006a,b). This ultimately yields the constraints imposed for the metal enrichment and dust production in the early epoch of the universe, (Jiang et al., 2016). The largest SMBH-candidates are a few $10^{10} M_{\odot}$ (McConnell et al., 2011, Postman et al., 2012, Scharẅachter et al., 2016, Thomas et al., 2016).

The first $z>5$ quasars were found in the the large-area Sloan Digital Sky Survey (SDSS, (Fan et al., 1999, 2001, Pâris et al., 2012, 2014, Schneider et al., 2010)). The SDSS quasar surveys provided the largest quasar sample $(\sim 500$ quasars at $z>4.5)$. Quasars at $z>4.5$ are routinely discovered in varieties of wide-field surveys, including the Canada-France High-Redshift Survey (CFHQS, (Willott et al., 2007)). There are newly identified quasars from the the Panoramic Survey Telescope and Rapid Response System (Pan-STARRS, (Bañados et al., 2018, Kaiser et al., 2002)), the UKIRT Infrared Deep Sky Survey (UKIDSS, (Lawrence, 2007, Yang et al., 2017)), Large Area Survey (ULAS), the VISTA VIKING (Edge, 2013), and VST ATLAS (Shanks, 2015) surveys, the Subaru Suprime Cam surveys (Kashikawa, 2015), and the Hyper Suprime Cam (HSC) survey (Matsuoka, 2016), the Dark Energy Survey (DES, (Reed et al., 2015)), and the Dark Energy Camera Legacy Survey (DECaLS, (Wang et al., 2007)).

However, the SDSS spectroscopic surveys have a lower degree of completeness at high redshift. The SDSS only covers a large portion of the Northern sky, although the Pan-STARRS1 survey (PS1, (Chambers, 2011, Kaiser et al., 2002, 2010)) has extended coverage of 1.5 hemispheres to a declination of -30 deg South. The Sky Mapper Southern Survey is a full hemispheric imaging survey carried out by the Sky Mapper telescope at Siding Spring Observatory in New South Wales, Australia (Wolf et al., 2018). By combining Wide-field Infrared Survey Explorer (WISE) and SDSS photometric data, Wang et al. (2016) have spectroscopically identified 72 new $z \sim 5.0$ quasars. The Table A1 is summing up the kinetic recession velocities of some typical distant astronomical objects with spectroscopic redshift determinations collected from the literature. 
Table 1. The kinetic recession velocities $\left(\beta_{r e c}\right)$ (in units of the speed of light) of some typical distant astronomical objects.

\begin{tabular}{|c|c|c|c|}
\hline Name & Redshift & Type (Ref.) & $\beta_{\text {rec }}$ \\
\hline GN-z11 & 11.09 & Galaxy, e.g. (1) & 0.819 \\
\hline MACS1149-JD1 & 9.11 & Galaxy, e.g. (2) & 0.807 \\
\hline EGSY8p7 & 8.68 & Galaxy, e.g. (3) & 0.804 \\
\hline A2744 YD4 & 8.38 & Galaxy, e.g. (4) & 0.801 \\
\hline GRB 090423 & 8.2 & Gamma-ray burst, e.g. (5) & 0.8 \\
\hline EGS-zs8-1 & 7.73 & Galaxy, e.g. (1) & 0.796 \\
\hline ULAS J1342+0928 & 7.54 & Quasar, e.g. (6) & 0.794 \\
\hline A1689-zD1 & 7.5 & Galaxy, e.g. (7) & 0.793 \\
\hline BDF-3299 & 7.109 & Galaxy, e.g. (8) & 0.789 \\
\hline ULAS J0109-3047 & 6.75 & Quasar, e.g. (9) & 0.785 \\
\hline ULAS J0305-3150 & 6.6 & Quasar, e.g. (9) & 0.783 \\
\hline HCM-6A & 6.56 & Galaxy, e.g. (10) & 0.783 \\
\hline CFHQS J2329-0301 & 6.417 & Quasar, e.g. (11) & 0.781 \\
\hline SDSS J010013.02+280225.8 & 6.3 & Quasar, e.g. $(12,13)$ & 0.78 \\
\hline SDSSJ1048+4637 & 6.2 & Quasar, e.g. $(14,15)$ & 0.778 \\
\hline SDSS J125051.93+313021.9 & 6.13 & Quasar, e.g. (16) & 0.777 \\
\hline SDSS J030331.40001912.9 & 6.07 & Quasar, e.g. $(17,18)$ & 0.777 \\
\hline CFHQS J1641+3755 & 6.047 & Quasar, e.g. (19) & 0.776 \\
\hline SDSS J081827.40+172251.8 & 6 & Quasar, e.g. $(15,16)$ & 0.776 \\
\hline PSO J183.2991-12.7676 & 5.86 & Quasar, e.g. (19) & 0.774 \\
\hline J104433.04-012502.2 & 5.8 & Quasar, e.g. (20) & 0.773 \\
\hline PSO J215.1514-16.0417 & 5.73 & Quasar, e.g. (19) & 0.772 \\
\hline PSO J045.1840-22.5408 & 5.7 & Quasar, e.g. (19) & 0.771 \\
\hline HDF 4-473.0 & 5.6 & Galaxy, e.g. (20) & 0.77 \\
\hline RD300 & 5.5 & Quasar, e.g. (21) & 0.768 \\
\hline SDSS J003125.86+071036.92 & 5.33 & Quasar, e.g. (13) & 0.765 \\
\hline SDSS J133257.45+220835.91 & 5.11 & Quasar, e.g. (13) & 0.761 \\
\hline SDSS J160111.16-182835.08 & 5.06 & Quasar, e.g. (13) & 0.76 \\
\hline SDSSp J120441.73-002149.6 & 5.03 & Quasar, e.g. $(22,23)$ & 0.76 \\
\hline SDSS J025121.33+033317.42 & 5 & Quasar, e.g. (13) & 0.759 \\
\hline PC $1247+3406$ & 4.9 & Quasar, e.g. (21) & 0.757 \\
\hline SDSS J014741.53-030247.88 & 4.75 & Quasar, e.g. (13) & 0.754 \\
\hline BR 1033-0327 & 4.51 & Quasar, e.g. (22) & 0.749 \\
\hline Q0046-293 & 4.01 & Quasar,e.g. (23) & 0.737 \\
\hline Q1208+1011 & 3.8 & Quasar,e.g. (24) & 0.731 \\
\hline OH471 & 3.408 & Quasar, (25) & 0.718 \\
\hline $4 \mathrm{C} 05.34$ & 2.877 & Quasar,e.g. (26) & 0.697 \\
\hline $5 \mathrm{C} 02.56$ & 2.399 & Quasar,e.g. (27) & 0.672 \\
\hline $3 \mathrm{C} 241$ & 1.617 & radio galaxy, e.g. (28) & 0.607 \\
\hline $3 \mathrm{C} 252$ & 1.105 & radio galaxy, e.g. (29) & 0.533 \\
\hline
\end{tabular}

(1) Oesch et al. (2016); (2) Hashimoto et al. (2018); (3) Zitrin et al. (2015); (4) Laporte et al. (2017); (5) Tanvir et al. (2009); (6) Bañados et al. (2018); (7) Watson et al. (2015); (8) Vanzella et al. (2011); (9) Venemans et al. (2013); (10) Hu et al. (2002); (11) Willott et al. (2010); (12) Wu et al. (2015); (13) Wang et al. (2016); (14) Fan et al. (2006b); (15) Fan et al. (2006c); (16) Wang et al. (2007); (17) Jiang et al. (2008); (18) Kurk et al. (2009); (19) Bañados et al. (2014); (20) Jiang et al.

(2007); (21) Stern et al. (2002); (22) Iwamuro \& Motohara (2002); (23) Warren et al. (1987);

(24) Schmidt et al. (1987); (25) Warren \& Hewett (1990); (26) Bahcall \& Oke (1971); (27) Lynds \&

Wills (1970); (28) Lilly \& Longair (1984); (29) Stern \& Spinrad (1999) 